\title{
Many-body partition function and thermal Hartree-Fock approximations
}

\author{
B. Farid \\ Max Planck Institute fur Festkorperforschung, Heisenbergstrasse 1, 70569 Stuttgart, Germany \\ N. H. March \\ Oxford University, Oxford, United Kingdom \\ and Department of Physics, University of Antwerp (RUCA), Groenenborgerlaan 171, B-2020, Belgium
}

A. K. Theophilou

Institute of Materials Science, Democritos NCSR, 15310 Athens, Greece

(Received 21 April 1998; revised manuscript received 13 March 2000)

\begin{abstract}
The hierarchy equations for the quantum thermal density matrices of an assembly of interacting particles in an external potential are derived in a compact form. This approach can complement and finally replace the phenomenological treatment of the thermodynamic properties of quantum gasses, liquids, and solids. We also derive a temperature Hartree-Fock approximation for fermions based on the symmetry properties of the exact hierarchy and finally we discuss the present approximation in relation to that of Kaplan and Argyres [T. A. Kaplan and P. N. Argyres, Ann. Phys. (N.Y.) 92, 1 (1975)].

PACS number(s): 05.30.- d
\end{abstract}

\section{INTRODUCTION}

Although all information about a system of $N$ identical particles is contained in the $N$-particle density matrix, many important physical quantities can be obtained from low-order density matrices. The particle density and the kinetic energy are important physical quantities, which can be calculated via the single-particle density matrix (DM), while for the total energy of a system with two particle interactions the two-particle DM is needed. Thus, it would be useful if one could set up equations for lower-order density matrices, ignoring the higher-order ones, as in most experiments only quantities involving one- and two-particle DM's are measured. In fact, many phenomenological equations, like, e.g., the Boltzmann equation and the diffusion equation, concern single-particle density matrices. Unfortunately, the low-order density-matrix equations derived from first principles, involve higher-order DM's and therefore one has to deal with a hierarchy of equations [1]. The way out is to terminate these hierarchies by expressing the higher-order reduced density matrices (RDM's) in terms of lower-order ones. This is in principle possible for the ground state and thermal equilibrium states, since the diagonal elements of the single-particle density matrix determine uniquely the higher-order density matrices. This is a corollary, directly derived from the Hohenberg and Kohn theorem [2] and its generalizations to thermodynamic systems [3].

In actual calculations concerning the thermal properties of quantum gases, liquids, and solids, one has to know the explicit forms of the functions which express the dependence of the high-order density matrices on the lower-order ones and thus it is necessary to use approximations. Then one can use the exact hierarchies to justify or improve already existing phenomenological equations describing these systems. Such an example is the Landau-Silin equation [4] set up phenomenologically to describe Fermi liquids such as helium-3. This equation was later derived from first prin- ciples by Glezos and Theophilou [5], while Brosens et al. [6] derived a similar equation in studying the dielectric properties of the electron gas for which many phenomenological equations existed before. These studies, however, concerned eigenstate properties and not thermodynamic equilibrium.

Another possible application of the thermal hierarchy equations is their use in the derivation of approximations for the thermal exchange and correlation energy density functional, $E_{\mathrm{xc}}(n)$, in the frame of a thermal density-functional theory (TDFT) [3]. Approximations concerning the groundstate density matrices hierarchy have already been obtained by Holas and March [7].

The present paper deals with the hierarchy of thermal density matrices of a system of $N$ identical particles in an external potential $V$, in thermodynamic equilibrium, interacting via two-body forces, i.e., the Hamiltonian is of the form

$$
H_{N}=\sum_{i=1}^{N} t_{i}+\sum_{i \neq j=1}^{N} t_{i, j} .
$$

For electrons that we focus on here, $t_{i, j}=e^{2} / r_{i j}$ is the Coulomb interaction between electrons at separation distance $r_{i j}$. Our objective is to use existing knowledge to construct a hierarchy for the reduced density matrices.

Let $\Psi_{\mathrm{Ni}}\left(x_{1}, x_{2}, \ldots, x_{N}\right)$ be an eigenfunction of the $N$-fermion Hamiltonian $H_{N}$, where the variable $x$ stands for the position and spin variable. Then, by multiplying both sides of the eigenvalue equation by $\Psi_{\mathrm{Ni}}^{*}\left(x_{1}^{\prime}, x_{2}^{\prime}, \ldots, x_{N}^{\prime}\right)$ one gets

$$
\begin{aligned}
& H_{N}\left(x_{1}, x_{2}, \ldots, x_{N}\right) n_{i}^{(N)}\left(x_{1}, \ldots, x_{N} ; x_{1}^{\prime}, x_{2}^{\prime}, \ldots, x_{N}^{\prime}\right) \\
& \quad=E_{\mathrm{Ni}} n_{i}^{(N)}\left(x_{1}, \ldots, x_{N} ; x_{1}^{\prime}, x_{2}^{\prime}, \ldots, x_{N}^{\prime}\right)
\end{aligned}
$$

where 


$$
\begin{aligned}
& n_{i}^{(N)}\left(x_{1}, x_{2}, \ldots, x_{N} ; x_{1}^{\prime}, x_{2}^{\prime}, \ldots, x_{N}^{\prime}\right) \\
& \quad=\Psi_{\mathrm{Ni}}\left(x_{1}, x_{2}, \ldots, x_{N}\right) \Psi_{\mathrm{Ni}}^{*}\left(x_{1}^{\prime}, x_{2}^{\prime}, \ldots, x_{N}^{\prime}\right)
\end{aligned}
$$

and the reduced single state density matrices are defined by the relation

$$
\begin{aligned}
n_{i}^{(B)} & \left(x_{1}, \ldots, x_{s} ; x_{1}^{\prime}, \ldots, x_{s}^{\prime}\right) \\
= & \left(\begin{array}{c}
N \\
S
\end{array}\right) \int d x_{s+1}, \ldots, d x_{N} \\
& \times n_{i}^{(N)}\left(x_{1}, \ldots, x_{s}, x_{s+1}, \ldots, x_{N} ; x_{1}^{\prime}, x_{2}^{\prime}, \ldots, x_{s}^{\prime} x_{s+1}, \ldots, x_{N}\right) .
\end{aligned}
$$

The integration sign in the following will imply that one has to equate primed and unprimed integration variables before integrating.

After successive integration of both sides of Eq. (2a), one gets the hierarchy of equations for the reduced density matrices $n_{k}^{(s)}$ :

$$
\begin{aligned}
& H_{s} n_{k}^{(s)}+(s+1) \int d x_{s+1}\left(t_{s+1}+\sum_{i=1}^{s} t_{i, s+1}\right) n_{k}^{(s+1)}+(s+1) \\
& \times(s+2) / 2 \int d x_{s+1} d x_{s+2} t_{s+1, s+2} n_{k}^{(s+2)}=E_{N k} n_{k}^{(s)}, \quad(3 \mathrm{a})
\end{aligned}
$$

where

$$
H_{s}=\sum_{i=1}^{s} t_{i}+\sum_{i \neq j=1}^{s} t_{i, j}, \quad H_{1}=t_{1} .
$$

For deriving these equations, we followed the procedure of Cohen and Frishberg [1] and we have also adopted their notation with minor changes to embrace excited states.

\section{DERIVATION OF THE THERMODYNAMIC HIERARCHY EQUATIONS} is [8]

The $N$-particle canonical ensemble Bloch density matrix

$$
\begin{aligned}
C^{(N)} & \left(x_{1}, \ldots, x_{s}, \ldots, x_{N} ; x_{1}^{\prime}, x_{2}^{\prime}, \ldots, x_{s}^{\prime}, \ldots, x_{N}^{\prime}, \beta\right) \\
= & \sum_{i} \exp \left(-\beta E_{1}\right) \\
& \times n_{i}^{(N)}\left(x_{1}, x_{2}, \ldots, x_{N} ; x_{1}^{\prime}, x_{2}^{\prime}, \ldots, x_{N}^{\prime}\right) .
\end{aligned}
$$

The definition of the lower-order reduced Bloch density matrices $C^{(s)}$ is $[8,9]$

$$
\begin{aligned}
C^{(s)} & \left(x_{1}, x_{2}, \ldots, x_{s} ; x_{1}^{\prime}, x_{2}^{\prime}, \ldots, x_{s}^{\prime}\right) \\
= & \left(\begin{array}{c}
N \\
S
\end{array}\right) \int d x_{s+1}, \ldots, d x_{N} \\
& \times C^{(N)}\left(x_{1}, x_{2}, \ldots, x_{N} ; x_{1}^{\prime}, x_{2}^{\prime}, \ldots, x_{N}^{\prime}\right),
\end{aligned}
$$

i.e., they are derived from the higher-order ones by progressively integrating out information. Integrating over all variables, one gets the many-body partition function for a canonical ensemble,

$$
\begin{gathered}
Z(\beta)=N^{-1} \sum_{i} \exp \left(-\beta E_{i}\right) \int d x n_{i}^{(1)}\left(x, x^{\prime}\right) \text { or } \\
Z(\beta)=\operatorname{Tr}\{\exp (-\beta H)\},
\end{gathered}
$$

by means of which the macroscopic properties of a system in thermodynamic equilibrium can be derived. The Bloch DM's differ from the thermal DM's only by the multiplicative factor $Z(\beta)$, i.e., $C^{(s)}=Z(\beta) n^{(s)}$.

The reduced single-particle density matrix is identical to the single-particle temperature Green's function $G\left(x^{\prime}, t^{\prime} ; x, t ; \beta\right)$ in the limit that $t^{\prime}-t=\varepsilon \rightarrow 0, \varepsilon>0$ [10]. This function is defined in terms of the fermion field operators $\hat{\psi}\left(x^{\prime}, t^{\prime}\right), \hat{\psi}^{\dagger}(x, t)$ by the relation

$$
G\left(x^{\prime}, t^{\prime}, x t ; \beta\right)=\operatorname{Tr}\left\{\exp (-\beta H-\mu N) T\left[\hat{\psi}\left(x^{\prime}, t^{\prime}\right) \hat{\psi}^{\dagger}(x, t)\right]\right\},
$$

where $T$ is the time-ordering operator. Similar relations hold for the higher-order Bloch density matrices and the corresponding Green's functions [10]. Thus in general thermodynamic Green's functions contain more information than the corresponding density matrices as they involve the time variable.

By using Eq. (4) it is straightforward to derive the relation

$$
C^{(s)}=\sum_{i} \exp \left(-\beta E_{i}\right) n_{i}^{(s)}, \quad 1 \leqslant s \leqslant N
$$

which will be used for the setting up of the hierarchy equations for the reduced density matrices. We multiply Eq. (3a) throughout by $\exp \left(-\beta E_{k}\right)$ and sum over the entire level spectrum $k$. Then, employing the same notation as in Eq. (3) we can write

$$
\begin{aligned}
& H_{s} C^{(s)}+(s+1) \int d x_{s+1}\left(t_{s+1}+\sum_{i=1}^{s} t_{i, s+1}\right) \\
& \quad \times C^{(s+1)}+[(s+1)(s+2) / 2] \\
& \quad \times \int d x_{s+1} d x_{s+2} t_{s+1, s+2} C^{(s+2)}=-\frac{\partial}{\partial \beta} C^{(s)},
\end{aligned}
$$

where the sum $\sum E_{k} \exp \left(-\beta E_{k}\right) n_{k}^{(s)}$ on the right has been replaced by $-(\partial / \partial \beta) C^{(s)}$. Thus we have derived the desired hierarchy equations. The energy levels $E_{i}$ and the corresponding wave functions do not appear in the final set of the hierarchy. This is expected to be so as one could use the trace definition of the density matrices and derive these equations without making any use of the energy eigenstates. This derivation is given in Appendix A for the one-particle reduced density matrix. In fact, the use of traces instead of eigenfunctions is more rigorous, as a Hamiltonian may not possess a complete set of eigenfunctions. However, we have chosen the presentation in terms of eigenfunctions as this is more comprehensible and is closely related to the groundstate derivation of Cohen and Frishberg [1]. It is worthwhile 
to observe that by writing the equation in terms of the normalized thermal density matrices $n\left(x_{1}, x_{1}^{\prime} ; \beta\right)$ $=C\left(x_{1}, x_{1}^{\prime} ; \beta\right) / Z(\beta)$ one gets a more complex equation.

\section{SYMMETRY PROPERTIES OF THE DENSITY MATRICES}

The differential operators in Eq. (7) are similar in form to those of the time-dependent Schrödinger equation, with $t$ $=-i \beta$, and Eq. (7) is covariant with respect to $I_{3}^{3}$, the group of all translations and rotations in the three-dimensional Euclidean space. This means that translation and rotation of the external potential gives a density-matrix solution with corresponding translation and rotation, in agreement with the corresponding property which follows directly from the definition of the $N$-particle DM's. Further, one can easily verify that all symmetry properties of the Hamiltonian are transferred to the one- and two-particle operators of Eq. (7). This is a direct consequence of the fact that integration over all space does not change the symmetry properties. Only the number of variables is reduced.

One can solve Eq. (7) starting from the zero-temperature $(\beta=\infty)$ DM's. In this case the transformation properties of the ground-state DM's related to the symmetry properties of the Hamiltonian will be transferred to the finite-temperature $\mathrm{N}$-particle DM and thereby to the lower-order ones. These properties are important and have to be imposed on the solutions in order to get a correct description of the physical system. Thus in the case of fermions the trace is over the space of antisymmetric wave functions while in the case of bosons one has to choose the space of symmetric ones. We summarize these properties below.

(a) The Bloch DM's are real and symmetric with respect to the exchange of primed and unprimed variables, i.e.,

$$
\begin{aligned}
C^{(s)} & \left(x_{1}, \ldots, x_{i}, \ldots, x_{k}, \ldots, x_{s} ; x_{1}^{\prime}, x_{i}^{\prime}, \ldots, x_{k}^{\prime}, \ldots, x_{s}^{\prime} ; \beta\right) \\
& =C^{(s)}\left(x_{1}^{\prime}, \ldots, x_{i}^{\prime}, \ldots, x_{k}^{\prime}, \ldots, x_{s}^{\prime} ; x_{1}, \ldots, x_{i}, \ldots, x_{k}, \ldots, x_{s} ; \beta\right) .
\end{aligned}
$$

This property is a direct consequence of the definition when one chooses real wave functions (in the case of degeneracy one can also use a real basis). In order to see the importance of this condition, take the case of the equation of the $N$-particle DM. As the differential operators in Eq. (7) affect only the primed variables, one would have the freedom to choose any unprimed part, if the symmetry constraint (a) is not imposed to the solutions.

(b) It is essential to notice that the solutions of Eq. (7) belong to the irreducible representations (Irreps) of the permutation group of the indices for the unprimed and primed variables separately. In the case of fermions, the antisymmetry Irrep must be chosen, i.e., the density matrix must satisfy the following relation:

$$
\begin{aligned}
& C^{(s)}\left(x_{1}, \ldots, x_{i}, \ldots, x_{k}, \ldots, x_{s} ; x_{1}^{\prime}, \ldots, x_{i}^{\prime}, \ldots, x_{k}^{\prime}, \ldots, x_{s}^{\prime} ; \beta\right) \\
& \quad=-C^{(s)}\left(x_{1}, x_{k}, \ldots, x_{i}, \ldots, x_{s} ; x_{1}^{\prime}, \ldots, x_{i}^{\prime}, \ldots, x_{k}^{\prime}, \ldots, x_{s}^{\prime} ; \beta\right) .
\end{aligned}
$$

The same relation holds for the primed variables. (c) For many-electron systems in an external potential $V(x)$, the density matrices have the same symmetry as the external potential. Thus, let the external potential have the property $V(g x)=V(x)$, where $g$ is a rotation or translation belonging to a group $G$. As $G$ is a subgroup of $I_{3}^{3}$, it follows that the Hamiltonian $H$ is also invariant under $G$, as the kinetic and interaction energy term of $H$ is invariant under $I_{3}^{3}$, which contains $G$. We shall show that

$$
\begin{array}{r}
C^{(s)}\left(g x_{1}, \ldots, g x_{s} ; g x_{1}^{\prime}, \ldots, g x_{s}^{\prime} ; \beta\right) \\
=C^{(s)}\left(x_{1}, \ldots, x_{s} ; x_{1}^{\prime}, \ldots, x_{s}^{\prime} ; \beta\right) .
\end{array}
$$

The proof is based on the trace property $\operatorname{Tr}\{A B\}$ $=\operatorname{Tr}\{B A\}$. Thus

$$
\operatorname{Tr}\left\{e^{-\beta H} g n g^{-1}\right\}=\operatorname{Tr}\left\{g^{-1} e^{-\beta H} g n\right\} .
$$

By taking into account that $g$ commutes with $H$ and therefore with $e^{-\beta H}$, we get Eq. (10). Thus, e.g., the thermal average of the electron density in a solid will have the space-group symmetry of its lattice.

The hierarchy equations were used in [7] for the derivation of an approximation for the exchange and correlation potential $V_{\mathrm{xc}}(n)$ for the case of the ground state. Obviously one can use the present hierarchy of density matrices to derive an approximation in the frame of a thermal density functional formulation, like the one established by Mermin [4]. In such an approximations, $V_{\mathrm{xc}}(n)$ will have the symmetry of the density and therefore of the external potential. This is not the case when one considers a density-functional approach for single states, as degeneracy may give rise to density asymmetries, which are transmitted to $V_{\mathrm{xc}}(n)$ [11].

If the Hamiltonian has an additional symmetry beyond that of the permutations of the indices, one can classify the energy eigenstates according to the Irreps of the symmetry group, and consider traces in a subspace with states of a definite Irrep. In this way one can develop reduced DM hierarchies labeled by the Irreps of the symmetry group. Such DM's are important in problems of broken ergodicity [12]. As the derivation is as that of the general case, with the space of states restricted, we shall not repeat the proof. The hierarchy obtained is as that of Eq. (7) with the reduced density matrices carrying an additional index, labeling the Irrep. Then a density matrix defined by Eqs. (4) is the $s$ sum over all matrices labeled by the Irreps of the symmetry group. Thus, e.g., one can label the density matrices by the spin index $S$ when no spin-orbit coupling is considered and no magnetic field is present.

\section{THERMODYNAMIC APPROXIMATIONS FOR FERMION SYSTEMS}

The simplest approximation for the ground-state theory, which takes into account the fermionic character of the particles, is the Hartree-Fock (HF) approximation. The hierarchy equations for this approximation were derived by Cohen and Frishberg [1]. As for the thermodynamic HF, we have two versions: the thermal HF (THFA) and the single determinant $\mathrm{HF}\left(T_{\mathrm{SDA}}\right)$ derived and discussed in detail by Kaplan and Argyres [13]. The first one considers an approximate density operator of the form $\exp \left[-\beta\left(K\left(n_{i}\right)-\mu N\right)\right]$, where $K$ 
is a linear function of the particle number operators $n_{i}$ while the other is free of this restriction. In both cases the grandcanonical potential is minimized with respect to this density approximation.

In the present paper, we develop an approximation based on the simplest symmetry-preserving expression of the DM's. Thus for fermions we write

$$
C^{(s)}\left(x_{1}, x_{2}, \ldots, x_{s} ; x_{1}^{\prime}, x_{2}^{\prime}, \ldots, x_{s}^{\prime} ; \beta\right)=s !^{-1} \operatorname{det}\left[C\left(x_{i} x_{j}^{\prime} ; \beta\right)\right],
$$

where by the bracket [] we denote the $s$ by $s$ matrix, with matrix elements $C_{i j}=C\left(x_{i}, x_{j}^{\prime}\right)$ and the approximation to $C^{(1)}$ is denoted by $C$. This is the simplest expression for $C^{(s)}$ having the property of antisymmetry with respect to the exchange of two primed or unprimed variables. The present approximation is similar to that of the ground-state theory of Cohen and Frishberg and as is well known it does not hold for densities of the form $\exp (-\beta H)$ when $H$ involves twoparticle operators. In order to avoid confusion with the exact DM's, we shall consider that the above thermal averages hold for an approximate statistical density operator, which in the following will be denoted by $\exp (-\beta K)$, where the approximate Hamiltonian $K$ involves only single-particle interactions. By introducing in Eq. (7) the decoupling scheme of Eq. (11), for the case $s=1$, we get the equation for $C$. After summing the various terms resulting from the expansion of the determinants, the following integrodifferential equation is obtained with $U\left(x_{1}, x_{2}\right)$ denoting the two-particle interaction:

$$
\begin{aligned}
-\frac{\partial}{\partial \beta} C\left(x_{1}, x_{1}^{\prime} ; \beta\right)= & {\left[-\nabla_{1}^{2} / 2+V\left(x_{1}\right)\right] C\left(x_{1} x_{1}^{\prime} ; \beta\right)+V_{C b}\left(x_{1} ; \beta\right) C\left(x_{1}, x_{1}^{\prime} ; \beta\right)-\int d x_{2} U\left(x_{1}, x_{2}\right) C\left(x_{1} x_{2} ; \beta\right) C\left(x_{2}, x_{1}^{\prime} ; \beta\right) } \\
& +\left[E(\beta)+E_{c b}(\beta)-E_{\mathrm{xc}}(\beta)\right] C\left(x_{1}, x_{1}^{\prime} ; \beta\right)-\int d x_{2}\left[\left(-\nabla_{2}^{\prime}\right)^{2} / 2+V\left(x_{2}^{\prime}\right)\right] C\left(x_{1}, x_{2}^{\prime} ; \beta\right) C\left(x_{2}, x_{1}^{\prime} ; \beta\right) \\
& -\int d x_{2} V_{C b}\left(x_{2} ; \beta\right) C\left(x_{1}, x_{2} ; \beta\right) C\left(x_{2}, x_{1}^{\prime} ; \beta\right)-\iint d x_{2} d x_{3} U\left(x_{2}, x_{3}\right) C\left(x_{1} ; x_{2} ; \beta\right) C\left(x_{2}, x_{1}^{\prime} ; \beta\right) \\
& \times C\left(x_{3} ; x_{3} ; \beta\right)+\iint d x_{2} d x_{3} U\left(x_{2} x_{3}\right) C\left(x_{1} ; x_{2} ; \beta\right) C\left(x_{2} x_{3} ; \beta\right) C\left(x_{3}, x_{1}^{\prime} ; \beta\right),
\end{aligned}
$$

where

$$
V_{C b}\left(x_{1} ; \beta\right)=\int d x_{2} U\left(x_{1}, x_{2}\right) C\left(x_{2}, x_{2} ; \beta\right)
$$

is the two-particle direct (Hartree) potential, and

$$
\begin{aligned}
E(\beta)=2^{-1} \int d x_{2}\left[-\nabla_{2}^{2} / 2+V\left(x_{2}\right)\right] C\left(x_{2}, x_{2} ; \beta\right), \\
E_{c b}(\beta)=2^{-1} \iint d x_{2} d x_{3} U\left(x_{2}, x_{3}\right) C\left(x_{2} ; x_{2} ; \beta\right) \\
\quad \times C\left(x_{3}, x_{3} ; \beta\right), \\
E_{\mathrm{xc}}(\beta)=2^{-1} \iint d x_{2} d x_{3} U\left(x_{2}, x_{3}\right) C\left(x_{2} ; x_{3} ; \beta\right) \\
\quad \times C\left(x_{3}, x_{2} ; \beta\right) .
\end{aligned}
$$

In all cases, before the integration is carried out the primed variable of integration is equated to the unprimed one. Note also the symmetry relations between primed variables which allowed us to use unprimed variables for the differential operators and the external potential.

Thus finally we have an equation in compact form, where no energy levels and eigenstates appear, which is an advantage with respect to the conventional thermodynamic approximation, which is expressed in terms of the eigenstates of a Hartree-Fock operator. We note that all symmetry prop- erties of the density matrix are preserved in this approximation. As this equation is of first order in $\beta$, one needs the initial condition. By calculating the zero-temperature $(\beta$ $=\infty$ ) reduced density matrix from the ground-state theory, one can find the solution at finite temperature. One can change variables to the center of mass $X=\left(x_{1}+x_{1}^{\prime}\right) / 2$ and relative positions variable $x=x_{1}-x_{1}^{\prime}$ and take the Fourier transform with respect to the $X$ variable. In this way one gets an equation with variables $x$ and $P$, where $P$ can be interpreted as the total momentum variable and $\nabla_{x} C(x ; P)$ as the diffusion term. The physical meaning of the external potential term can be seen when we consider small $x$. Then by expanding about $X$, one gets a force term $x \nabla V(X)$, affecting the relative motion. This motion is also affected by the direct and exchange potentials arising from the interaction term.

When the external potential goes to a constant, a solution, which depends only on $x$, is possible, provided that stable solutions of the equation exist. This depends on the form of the interaction potential $U\left(x_{I}, x_{j}\right)$. In the case of the electron gas, stability is not possible, as the interaction is repulsive. Then one has to consider the jellium model where the Hartree potential is canceled out by the positive background potential.

\section{DISCUSSION AND SUMMARY}

We shall first derive from Eqs. (12) an eigenfunction equation in order to compare it to that obtained by Cohen 
and Frishberg. Second, we shall prove that our thermodynamic HF approximation is related to the THFA rather than the TSDA of Kaplan and Argyres [13]. Our proof is based on the fact that the relation $\left\langle n_{i} n_{j}\right\rangle_{T}=\left\langle n_{i}\right\rangle_{T}\left\langle n_{j}\right\rangle_{T}$ does not hold in the TSDA whereas in the THFA it does. We first notice that $C$ is a Hermitian operator as $C\left(x, x^{\prime} ; \beta\right)=C\left(x^{\prime}, x ; \beta\right)$ and therefore it can be expanded in terms of a complete set of functions with only diagonal terms, i.e.,

$$
C\left(x, x^{\prime} ; \beta\right)=\sum_{i} n_{i} \varphi_{i}(x) \varphi_{i}\left(x^{\prime}\right)
$$

where both the wave functions and the coefficients depend on $\beta$. We shall take the $\varphi_{i}(x)$ as real, since in case $\varphi_{i}(x)$ is complex, its complex conjugate has the same eigenvalue. By making use of the orthogonality relation of the $\varphi_{i}(x)$, one finds

$$
\int d x^{\prime} C\left(x, x^{\prime} ; \beta\right) \varphi_{i}\left(x^{\prime}\right)=n_{i} \varphi_{i}(x)
$$

We next multiply both sides of Eq. (12) by $\varphi_{i}\left(x_{1}^{\prime}\right)$ and integrate over $x_{1}^{\prime}$. After taking into account Eqs. (13), we get

$$
\begin{aligned}
\int d x_{1}^{\prime} \varphi_{i}\left(x_{1}^{\prime}\right)\left(-\frac{\partial}{\partial \beta}\right) C\left(x_{1}, x_{1}^{\prime} ; \beta\right)= & \left.-\frac{1}{2} \nabla_{1}^{2}+V\left(x_{1}\right)\right] n_{i} \varphi_{i}\left(x_{1}\right)+V_{C b}\left(x_{1} ; \beta\right) n_{i} \varphi_{i}\left(x_{1}\right) \\
& -\int d x_{2} U\left(x_{1}, x_{2}\right) C\left(x_{1}, x_{2} ; \beta\right) n_{i} \varphi_{i}\left(x_{2}\right)+\left[E(\beta)+E_{c b}(\beta)-E_{\mathrm{xc}}(\beta)\right] n_{i} \varphi_{i}\left(x_{1}\right) \\
& -\int d x_{2}\left[\left(-\nabla_{2}^{\prime}\right)^{2} / 2+V\left(x_{2}^{\prime}\right)\right] C\left(x_{1}, x_{2}^{\prime} ; \beta\right) n_{i} \varphi_{i}\left(x_{2}\right) \\
& -\int d x_{2} V_{C b}\left(x_{2} ; \beta\right) C\left(x_{1}, x_{2} ; \beta\right) n_{i} \varphi_{i}\left(x_{2}\right) \\
& -\iint d x_{2} d x_{3} U\left(x_{2}, x_{3}\right) C\left(x_{1} ; x_{2} ; \beta\right) C\left(x_{3}, x_{3} ; \beta\right) n_{i} \varphi_{i}\left(x_{2}\right) \\
& +\iint d x_{2} d x_{3} U\left(x_{2}, x_{3}\right) C\left(x_{1} ; x_{2} ; \beta\right) C\left(x_{2}, x_{3} ; \beta\right) n_{i} \varphi_{i}\left(x_{3}\right),
\end{aligned}
$$

where one can recognize the HF operator on the right-hand side of the first two lines. Thus we get an equation which on the right-hand side is similar in form to that obtained by Cohen and Frishberg in [1]. To verify this, one can introduce in Eq. (14) the single-particle DM eigenfunction expansion given by Eq. (13a). Note that in the present case, the lefthand side is different and cannot be simplified since the $\varphi_{i}(x)$ depend on $\beta$.

In order to show that our equation is related to the THFA, we use our approximate $C^{(2)}$. Then according to the decoupling scheme of Eq. (11),

$$
\begin{aligned}
2 C^{(2)}\left(x_{1}, x_{2} ; x_{1}^{\prime}, x_{2}^{\prime} ; \beta\right)= & C\left(x_{1}, x_{1}^{\prime} ; \beta\right) C\left(x_{2}, x_{2}^{\prime} ; \beta\right) \\
& -C\left(x_{1}, x_{2}^{\prime} ; \beta\right) C\left(x_{2}, x_{1}^{\prime} ; \beta\right),
\end{aligned}
$$

where by $C^{(2)}$ we denote the approximate two-particle DM's. It is useful to see that in the explicit expression of $C^{\prime(2)}$, the $i=j$ terms vanish and therefore the above expression becomes

$$
\begin{aligned}
2 C^{2}\left(x_{1}, x_{1}^{\prime} ; x_{2}, x_{2}^{\prime} ; \beta\right)= & \sum_{i \neq j} n_{i} n_{j}\left\{\varphi_{i}\left(x_{1}\right) \varphi_{i}\left(x_{1}^{\prime}\right) \varphi_{j}\left(x_{2}\right) \varphi_{j}\left(x_{2}^{\prime}\right)\right. \\
& \left.-\varphi_{i}\left(x_{1}\right) \varphi_{i}\left(x_{2}^{\prime}\right) \varphi_{j}\left(x_{2}\right) \varphi_{j}\left(x_{1}^{\prime}\right)\right\} .
\end{aligned}
$$

We next multiply both sides of Eq. (15a) by $\varphi_{j}\left(x_{2}^{\prime}\right) \varphi_{i}\left(x_{1}^{\prime}\right)$ and integrate both sides. After integration we take into account Eq. (13b) and obtain

$$
\begin{gathered}
\int d x_{2}^{\prime} \varphi_{j}\left(x_{2}^{\prime}\right) \int d x_{1}^{\prime} \varphi_{i}\left(x_{1}^{\prime}\right) C^{(2)}\left(x_{1}, x_{2} ; x_{1}^{\prime}, x^{\prime} ; \beta\right) \\
=\left\langle n_{i}\right\rangle_{T}\left\langle n_{j}\right\rangle_{T}\left[\varphi_{i}\left(x_{1}\right) \varphi_{j}\left(x_{2}\right)-\varphi_{j}\left(x_{1}\right) \varphi_{i}\left(x_{2}\right)\right] .
\end{gathered}
$$

In order to show that the THFA relation

$$
\left\langle n_{i} n_{j}\right\rangle_{T}=\left\langle n_{i}\right\rangle_{T}\left\langle{ }_{j}\right\rangle_{T}
$$

holds, we have to use the second quantization representation of $C^{(2)}$. This is done in Appendix B.

We have compared and contrasted the present approach, based mainly on symmetry principles and the densities, to the THFA and TSDA of Kaplan and Argyres [13]. These authors focused attention, via the minimum principle for the grand-canonical potential. Their results on the THFA are posed in terms of one-electron eigenfunctions of a temperature-dependent $\mathrm{HF}$ operator and their corresponding eigenvalues. However, our objective in the present investigation has been to obtain an equation in compact form, independent of wave functions and energies, which by further simplifications could eventually lead to the first-principle derivation of already existing phenomenological equations, like, e.g., the stationary (time-independent) Boltzmann equa- 
tion. Further, our approach cannot give higher accuracy than the TSDA, since the Kaplan-Argyres approach is based on the best statistical density operator, which minimizes the grand-canonical potential. The derivation of the TSDA approximation in compact form would also be interesting and useful. The advantage of our derivation with respect to that of the method of Green's functions is that the time ordering which makes the equations more complicated is not present in our case.

To conclude, we consider that more insight is gained when the same approximation is seen from a different perspective.

\section{ACKNOWLEDGMENT}

We thank Professor A. Holas (Warsaw) for many valuable discussions in the general area of this investigation.

\section{APPENDIX A}

We shall derive the equation for $C\left(x, x^{\prime}\right)$ without making use of the eigenfunction expansion. We shall also use field operators. We write the Hamiltonian

$$
H=T+H_{\text {int }}+\hat{V}
$$

in terms of the fermion field operators $\hat{\psi}^{+}(\mathbf{x})$ and $\hat{\psi}(\mathbf{x})$, where the various terms of the Hamiltonian assume integral forms, namely

$$
\begin{aligned}
& T=2^{-1} \int d^{3} x \nabla \hat{\psi}^{+}(\mathbf{x}) \nabla \hat{\psi}(\mathbf{x}), \quad \hat{V}=\int \hat{n}(\mathbf{x}) V(\mathbf{x}) d^{3} x, \\
& H_{\mathrm{int}}=2^{-1} \int d^{3} x^{\prime} \int d^{3} x \hat{\psi}^{+}(\mathbf{x}) \hat{n}\left(\mathbf{x}^{\prime} \mathbf{x}\right) \hat{\psi}(\mathbf{x}) U\left(\left|\mathbf{x}, \mathbf{x}^{\prime}\right|\right) .
\end{aligned}
$$

The $\hat{\psi}^{+}(\mathbf{x}), \hat{\psi}(\mathbf{x})$ obey the anticommutation relations $\left[\hat{\psi}^{+}(\mathbf{x}), \hat{\psi}\left(\mathbf{x}^{\prime}\right)\right]_{+}=\delta\left(\mathbf{x}-\mathbf{x}^{\prime}\right)$ and the $\hat{n}\left(\mathbf{x}, \mathbf{x}^{\prime}\right)$ takes the simple form

$$
\hat{\mathbf{n}}\left(\mathbf{x}, \mathbf{x}^{\prime}\right)=\hat{\boldsymbol{\psi}}^{+}(\mathbf{x}) \hat{\boldsymbol{\psi}}\left(\mathbf{x}^{\prime}\right) .
$$

Then $n\left(\mathbf{x}, \mathbf{x}^{\prime} ; \beta\right)=\operatorname{Tr}\left\{\hat{\psi}^{+}(\mathbf{x}) \hat{\psi}\left(\mathbf{x}^{\prime}\right) \exp (-\beta H)\right\}$ and

$$
-\frac{\partial}{\partial \beta} C\left(\mathbf{x}, \mathbf{x}^{\prime} ; \beta\right)=\operatorname{Tr}\left\{\hat{\psi}^{+}(\mathbf{x}) \hat{\psi}\left(\mathbf{x}^{\prime}\right) H \exp (-\beta H)\right\} .
$$

We next make use of the relations

$$
\begin{gathered}
\hat{\psi}\left(\mathbf{x}^{\prime}\right) H=\left[\hat{\psi}\left(\mathbf{x}^{\prime}\right), H\right]+H \hat{\psi}\left(\mathbf{x}^{\prime}\right), \\
{\left[\hat{\psi}\left(\mathbf{x}^{\prime}\right), H\right]=\left[\hat{\psi}\left(\mathbf{x}^{\prime}\right), T\right]+\left[\hat{\psi}\left(\mathbf{x}^{\prime}\right), H_{\mathrm{int}}\right]+\left[\hat{\psi}\left(\mathbf{x}^{\prime}\right), V\right],} \\
{\left[\hat{\psi}\left(\mathbf{x}^{\prime}\right), T\right]=-2^{-1} \nabla^{2} \hat{\psi}\left(\mathbf{x}^{\prime}\right),} \\
{\left[\hat{\psi}\left(\mathbf{x}^{\prime}\right), H_{\mathrm{int}}\right]=\int d^{3} x^{\prime \prime} \hat{n}\left(\mathbf{x}^{\prime}, \mathbf{x}^{\prime \prime}\right) U\left(\mid \mathbf{x}^{\prime \prime}, \mathbf{x}^{\prime}\right) \hat{\psi}\left(\mathbf{x}^{\prime}\right)}
\end{gathered}
$$

and

$$
\left[\hat{\psi}\left(\mathbf{x}^{\prime}\right), \hat{V}\right]=V\left(\mathbf{x}^{\prime}\right) \psi\left(\mathbf{x}^{\prime}\right)
$$

After substituting the above relations in Eq. (A2b), we get

$$
\begin{aligned}
-\frac{\partial}{\partial \beta} n\left(\mathbf{x}, \mathbf{x}^{\prime} ; \beta\right)= & \operatorname{Tr}\left\{\hat{\psi}^{+}(\mathbf{x})\left[-2^{-1} \nabla^{\prime 2}+V\left(\mathbf{x}^{\prime}\right)\right]\right. \\
& +\int d^{3} x^{\prime \prime} U\left(\mid \mathbf{x}^{\prime \prime}, \mathbf{x}^{\prime}\right) \hat{\psi}^{+}(\mathbf{x}) \\
& \left.\times \hat{n}\left(\mathbf{x}^{\prime}, \mathbf{x}^{\prime \prime}\right) \hat{\psi}\left(\mathbf{x}^{\prime}\right) \exp (-\beta H)\right\} \\
& +\operatorname{Tr}\left\{\hat{\psi}^{+}(\mathbf{x}) H \hat{\psi}\left(\mathbf{x}^{\prime}\right) \exp (-\beta H)\right\} .
\end{aligned}
$$

Finally, by inserting the explicit form of $H$ in Eq. (A3a), noting that

$$
\begin{aligned}
\hat{\psi}^{+}(\mathbf{x}) & {\left[-2^{-1} \nabla^{\prime 2}+V\left(\mathbf{x}^{\prime}\right)\right] \hat{\psi}\left(\mathbf{x}^{\prime}\right) } \\
= & {\left[-2^{-1} \nabla^{\prime 2}+V\left(\mathbf{x}^{\prime}\right)\right] \hat{\psi}^{+}(\mathbf{x}) \hat{\psi}\left(\mathbf{x}^{\prime}\right) }
\end{aligned}
$$

and that the two-particle density matrix is

$$
\begin{aligned}
C^{2}\left(\mathbf{x}_{1}, \mathbf{x}_{2} ; \mathbf{x}_{1}^{\prime} \mathbf{x}_{2}^{\prime} ; \beta\right)= & 2^{-1} \operatorname{Tr}\left\{\hat{\psi}^{+}\left(\mathbf{x}_{1}\right) \hat{n}\left(\mathbf{x}_{2}, \mathbf{x}_{2}^{\prime}\right) \hat{\psi}\left(\mathbf{x}_{1}^{\prime}\right)\right. \\
& \times \exp (-\beta H)\}
\end{aligned}
$$

we get the hierarchy equation (7) for the first-order DM. Note that the term

$$
\operatorname{Tr}\left\{\hat{\psi}^{+}(\mathbf{x}) H_{\text {int }} \hat{\psi}\left(\mathbf{x}^{\prime}\right) \exp (-\beta H)\right\}
$$

involves third-order DM's.

\section{APPENDIX B}

We use Eq. (15) with $C^{(2)}\left(\mathbf{x}_{1}, \mathbf{x}_{2} ; \mathbf{x}_{1}^{\prime}, \mathbf{x}_{2}^{\prime} ; \beta\right)$ expressed in terms of the field operators as in Eq. (A3c), with the approximate Hamiltonian $K$ in place of $H$.

Then

$$
\begin{array}{rl}
\int d & d x_{2}^{\prime} \varphi_{j}\left(\mathbf{x}_{2}^{\prime}\right) \int d x_{1}^{\prime} \varphi_{i}\left(\mathbf{x}_{1}^{\prime}\right) \operatorname{Tr}\left\{\hat{\psi}^{+}\left(\mathbf{x}_{1}\right) \hat{\psi}^{+}\left(\mathbf{x}_{2}\right) \hat{\psi}\left(\mathbf{x}_{2}^{\prime}\right)\right. \\
& \left.\times \hat{\psi}\left(\mathbf{x}_{1}^{\prime}\right) \exp (-\beta K)\right\} \\
= & \left\langle\hat{n}_{i}\right\rangle_{T}\left\langle\hat{n}_{j}\right\rangle_{T}\left[\varphi_{i}\left(\mathbf{x}_{1}\right) \varphi_{j}\left(\mathbf{x}_{2}\right)-\varphi_{j}\left(\mathbf{x}_{1}\right) \varphi_{i}\left(\mathbf{x}_{2}\right)\right] .
\end{array}
$$

We next substitute the integrals of the primed variables of (B1) by the creation and annihilation operators defined as follows:

$$
\hat{a}_{i}^{+}=\int d^{3} x \varphi_{i}\left(\mathbf{x}_{1}\right) \hat{\psi}^{+}\left(\mathbf{x}_{1}\right), \quad \hat{a}_{i}=\int d^{3} x^{\prime} \varphi_{i}\left(\mathbf{x}_{1}\right) \hat{\psi}\left(\mathbf{x}_{1}\right)
$$

and the left-hand side of Eq. (B1) becomes 


$$
\begin{aligned}
\int d x_{2}^{\prime} \varphi_{j}\left(\mathbf{x}_{2}^{\prime}\right) \int d x^{\prime} \varphi_{i}\left(\mathbf{x}_{I}^{\prime}\right) \operatorname{Tr}\left\{\hat{\psi}^{+}\left(\mathbf{x}_{1}\right)\right. \\
\left.\times \hat{\psi}^{+}\left(\mathbf{x}_{2}\right) \hat{\psi}\left(\mathbf{x}_{2}^{\prime}\right) \hat{\psi}\left(\mathbf{x}_{1}^{\prime}\right) \exp (-\beta K)\right\} \\
=\operatorname{Tr}\left\{\hat{\psi}^{+}\left(\mathbf{x}_{1}\right) \hat{\psi}^{+}\left(\mathbf{x}_{2}\right) \hat{a}_{j} \hat{a}_{i} \exp (-\beta K)\right\} .
\end{aligned}
$$

By multiplying by $\varphi_{i}\left(x_{1}\right) \varphi_{j}\left(x_{2}\right)$, for $i \neq j$, on both sides of
Eq. (B1) and integrating we find after taking into account the orthogonality relations

$$
\operatorname{Tr}\left\{\hat{a}_{i}^{\dagger} \hat{a}_{j}^{\dagger} \hat{a}_{j} \hat{a}_{i} \exp (-\beta K)\right\}=\left\langle\hat{n}_{i}\right\rangle\left\langle\hat{n}_{j}\right\rangle_{T}, \quad \text { for } \quad i \neq j .
$$

We next take into account that for $i \neq j, \quad \hat{a}_{i}^{\dagger} \hat{a}_{j}^{\dagger} \hat{a}_{j} \hat{a}_{i}$ $=\hat{a}_{i}^{\dagger} \hat{a}_{i} \hat{a}_{j}^{\dagger} \hat{a}_{j}=\hat{n}_{i} \hat{n}_{j}$. Thus finally we find the desired relation $\operatorname{Tr}\left\{\left\{\hat{n}_{i} \hat{n}_{j} \exp (-\beta K)\right\}=\left\langle\hat{n}_{i}\right\rangle_{T}\left\langle\hat{r}_{j}\right\rangle_{T}\right.$ for $i \neq j$.
[1] L. Cohen and C. Frishberg, Phys. Rev. A 13, 927 (1976).

[2] P. Hohenberg and W. Kohn, Phys. Rev. 136, B864 (1964).

[3] N. D. Mermin, Phys. Rev. 137, A1441 (1965).

[4] L. Landau, Zh. Eksp. Teor. Fiz. 5, 69 (1957) [Sov. Phys. JETP 32, 58 (1957)].

[5] N. M. Glezos and A. K. Theophilou, Physica A 146, 360 (1987).

[6] F. Brosens, J. T. Devreese, and L. F. Lemmens, Phys. Rev. B 21, 1349 (1980); 21, 1363 (1980); Phys. Status Solidi B 74, 45 (1976).

[7] A. Holas and N. H. March, Phys. Rev. A 51, 2040 (1995); M. Levy and N. H. March, ibid. 55, 1885 (1997); A. Holas and N.
H. March, Int. J. Quantum Chem. 61, 263 (1997); A. Holas and M. Levy, Phys. Rev. A 56, 1031 (1997).

[8] N. H. March, W. H. Young, and S. Sampanthar, The Manybody Problem in Quantum Mechanics (Dover, New York, 1995).

[9] P. O. Loewdin, Phys. Rev. 97, 1474 (1955).

[10] A. L. Fetter and J. D. Valecka, Quantum Theory of ManyParticle Systems (McGraw Hill, New York, 1971).

[11] A. K. Theophilou, Int. J. Quantum Chem. 69, 461 (1998).

[12] R. G. Palmer, Adv. Phys. 31, 669 (1982).

[13] T. A. Kaplan and P. N. Argyres, Ann. Phys. (N.Y.) 92, 1 (1975). 